\title{
Direct Sowing of Tree Seeds Trial: Case Study of Kuani Hill in Meru County Kenya
}

\author{
Jesse Lugadiru ${ }^{1 *}$ Gordon Armstrong ${ }^{2}$, \\ 1. Kenya Forestry Research Institute -KEFRI Kenya box 2041200200 Nairobi Kenya \\ 2. Gordon Armstrong, DFID UK \\ * E-mail of the corresponding author: lugadiru@yahoo.com or jesselugadiru@gmail.com
}

\begin{abstract}
World climate change is as a result of greenhouse gases emitted into atmosphere. The gases remain in the atmosphere because of limited actively growing plants to sequestrate main culprit of greenhouse gases - carbon dioxide. This then calls for consolidated efforts to make trees start growing in a big number. Tree planting is expensive undertaking requiring funds and human energy. Some sites are so vast and others not accessible, this then requires simpler and less expensive mechanisms to do afforestation. An experiment was done to study possibilities of removing one stage in tree nurturing activities before transferring to planting spots. The nursery practice was removed which meant seeds are sown direct in ground and observation made. The aim of the trial was to attempt to establish trees by the direct sowing of seeds at planting site. If trees can be successfully established by this method then establishment costs would be greatly reduce by the elimination of nursery work. 12 different species were included in the trial. Some of the seeds required pretreatment which was done. There were two sowings in different seasons, the first was done when there were little rains and it failed prompting a repeat during next rains which were favorable. Results were, the first sowing was a failure with all germinated plants dying after sprouting. The second had some limited success with sprouting saplings surviving. This was limited success due to the site condition being semiarid. Among the best performers were drought resistance plants of Acacia albida (69\%), Albezia lebbek (8\%), Acacia aulacocarpa (3\%), Acacia auriculiformis (1\%), Senna siamea (1\%), and Gravellea robusta (1\%). The rest that is Acacia mearnsii, Acrocurpus fraxinfolius, Senna spectabilis, Eucalyptus globulus, Eucalyptus saligna and Leuceana leucocephala, scored zero percentage. The results indicate that direct sowing need to be done in high potential areas and if done in semiarid, then choose a good rainy season. Species to be used should match the site. This experiment need to be repeated in many different sites with many tree species.
\end{abstract}

Keywords: Tree Seeds Trial, Kenya

DOI: $10.7176 / \mathrm{JNSR} / 9-2-06$

\section{The location}

The trail is located on 1.2 hectares of land on northern side of Kuani hill which is a trust land administered by Meru county. The hill is adjacent to the main Meru- Mau road near Muthara village Tigania. Latitude is $0^{0} 13^{\prime}$ north and latitude is $37^{\circ} 47^{\prime}$ east. This can be found on survey of Kenya map 1:50,000 scale sheet 108/4, map reference CL 638237.

The site can be reached by a tarmac road to Isiolo which is off Meru-Mau road along foot of the hill. There are other tree trials also located on the same site.

\section{Site Description}

The site is on a steep slope (>37\%) north facing slope. Altitude is 1,500 meters above sea level. The soils are derived from volcanic lava and are described by Macharia and Kamotho of the Kenya soil Survey as follows;well drained, deep, dark reddish brown clay sols. They have ABC horizon sequence with diffuse, smooth to wavy soils. They have sub angular blocky structures. The top soil is severely over washed on the lower and middle parts of the site. The $\mathrm{pH}$ is 6.5. Details of soil are described in appendix 1

The natural vegetation of the hill is bush land with dominant shrubs being Combretum molle, Rhus vuilgaris, and Dodonea viscosa. Other shrubs are Acacia sp., Ziziphus sp., Cassia spp., Rhus natalensis, Piliostigma thoningii and Indofera sp. The dominant grasses are Cybopogen spp., hyprrhenia filipendula and Themeda triandra. There are natural trees and shrubs cover of $35 \%$ of the ground, grasses $55 \%$ and herbs $5 \%$.

Some parts of the hill have previously been planted at various times with Pinus patula, Eucalyptus Maculata and Gravellea robusta. Most of these plantings has been neglected and are ether suppressed or failed due to nonweeding, fires, browsing and drought. Many of the lower slopes of the hill are badly eroded with deep gullies and large landslips. Euphorbia turucalli has been planted across the gullies in an attempt to control erosion and large cut off drain dug around the summit. The tree planting and soil conservation measures were carried out 
under Rural development projects.

The hill was afforested again by Nyambene Tea Company and Kenya Forest Service mainly with Eucalyptus saligna, Eucalyptus globulus and some Eucalyptus maculata and Gravellea robusts.

\section{Climate}

The only rain gauge available was at Muthara tree nursery which is $13 \mathrm{~km}$ to northeast of the hill. Annual rain fall ranged from $670.1 \mathrm{~mm}$ to $803.5 \mathrm{~mm}$. The site falls in climatical zone IV-3.

\section{Site Preparation}

The only site preparation measures carried out before sowing was the digging of holes of pits then refilled at a spacing of $2.5 \mathrm{~m} \times 2.5 \mathrm{~m}$ according to the trial design. These pits were $30 \mathrm{~cm} \times 30 \mathrm{~cm}$ and $30 \mathrm{~cm}$ deep.

\section{Trial Design}

The trial was laid down in six replicates blocks aligned approximately parallel to contours of the hill. The upper three blocks (i-iii) are reasonably stable, ungullied land, whereas the lower three blocks (iv -vi) are on un stable, deep gullies land. Within each block, 16 tree plots $(4 \mathrm{x} 4)$ of each species are allocated at random and sowing $2.5 \mathrm{~m} \times 2.5 \mathrm{~m}$ spacing.

\section{Trial History}

The trial was first sown in the first rainy season in the year that is April4. The rains that season were very poor and most seeds failed to germinate. Those that did germinate did not survive for more than few months. Sowing was therefore repeated with exactly the same species in November rains which were good.

\section{Species}

The following species were included in the trial. The species seeds, of those requiring pre-treatments, (Acacia spp., Albezzia lebbek, Cassia spp. And Leuceana leucocephala) were treated immediately prior to sowing by immersing in hot water and leaving them to soak overnight in the cooling water.

Table 1. Shows species, seeds sources and number of seedlings put in each pit

\begin{tabular}{|l|l|l|l|l|}
\hline Code & Species & Provenance & $\begin{array}{l}\text { Seed } \\
\text { source }\end{array}$ & $\begin{array}{l}\text { No seeds } \\
\text { per pit }\end{array}$ \\
\hline PGG & Acacia albida & Not known & 4 & 2 \\
\hline GPR & Acacia aulacocarpa & Buckley (Bn.13865) & 3 & 1 \\
\hline GPY & Acacia auriculiformis & Springgvale Bn.13861 & 3 & 1 \\
\hline BPW & Acacia mearnsii & Nyambene & 1 & 4 \\
\hline PWB & Albezia lebbek & Not known & 4 & 3 \\
\hline PPR & Acrocurpus fraxinfolius & Embu & 1 & 4 \\
\hline WPG & Senna siamea & Ishiara & 1 & 4 \\
\hline WGP & Senna spectabilis & Embu & 1 & 4 \\
\hline BRY & Eucalyptus globulus & Meru & 1 & 10 \\
\hline X & Eucalyptus saligna & Mutghara & 1 & 10 \\
\hline Y & Gravellea robusta & Muthara & 1 & 4 \\
\hline GYP & Leuceana leucocephala & Ena & 1 & 4 \\
\hline
\end{tabular}

For seed sources see Appendix 1

\section{Results}

Initial germination was observed to be good for all species, but by time of one year after sowing that is following November, many of the seedlings had died and the percentage of pits containing surviving seedlings is as below table 2 
Table 2. Percent of pits containing surviving seedlings after one year of sowing (November 1985)

\begin{tabular}{|l|l|l|l|l|}
\hline & Code & Species & Provenance & $\begin{array}{l}\text { Percent pits with } \\
\text { seedlings (\%) }\end{array}$ \\
\hline 1 & PGG & Acacia albida & Not known & 69 \\
\hline 2 & GPR & Acacia aulacocarpa & Buckley (Bn.13865) & 3 \\
\hline 3 & GPY & Acacia auriculiformis & $\begin{array}{l}\text { Springgvale } \\
\text { Bn.13861 }\end{array}$ & 1 \\
\hline 4 & BPW & Acacia mearnsii & Nyambene & 0 \\
\hline 5 & PWB & Albezia lebbek & Not known & 8 \\
\hline 6 & PPR & Acrocurpus fraxinfolius & Embu & 0 \\
\hline 7 & WPG & Senna siamea & Ishiara & 1 \\
\hline 8 & WGP & Senna spectabilis & Embu & 0 \\
\hline 9 & BRY & Eucalyptus globulus & Meru & 0 \\
\hline 10 & X & Eucalyptus saligna & Muthara & 0 \\
\hline 11 & Y & Gravellea robusta & Muthara & 1 \\
\hline 12 & GYP & Leuceana leucocephala & Ena & 0 \\
\hline
\end{tabular}

\section{Conclusion}

The direct sowing heavily depend on rainfall otherwise there will be failure. The trail has shown some very limited success on this site. A drought resistance of specie of Acacia albida established very successfully. The growth rate of the seedling was extremely low compared to plant establishment using seedlings from the nursery.

\section{Discussions}

It is possible to do direct sowing of seeds of tree. From this experiment, there must be sufficient rains for good results. Very hash climatical zones are not appropriate for such undertaking. If a semi-arid land should be used then, drought resistance species should carefully be selected and used.

\section{Recommendations}

This experiment should be repeated elsewhere with comprehensive tree species and in wetter regions. There should be a comparison study between sites with soil loosen and those of no action with compact ground and weeds.

\section{References used}

1. Amstrong G.D., Lugadiru J.K. silvicultural Research programme, 1983-1986 -Embu -Meru -Isiolo Forestry Project, Kenya Forestry Project . Land Resourced Development Centre Tolworth Tower, Surbtion surrey KT6 7DY England UK.

2. Macharia P.N., Kimotho P.W., 1984 Soils and vegetation of Embu-Meru-Isiolo Forestry Projects trial sites. Kenya Soil Survey (site evaluation Report No. 70)

3. Chirchir R.K. 2000 Hill Reforestation : A case History of Kuani Hill Meru

\section{Appendix 1}

\section{Seed Sources}

1. Local collection

2. Kenya forestry research Institute

Po box 2041200200

Nairobi

3. C.S.I.R.O.

Division of Forestry Research

Po Box 4008

Queen Victoria Terrace

a.c.t. 2600

Australia

4. Timmers and layer

P.o. box 17

Heemstede 2100 AA

Holland

5. Chief Forest Research Officer

P.O. box 22099 
Kitwe

Zambia

6. Mr. Edmund Barrow

East Pokot Agriculture project

P. o. Malagat

\section{Appendix 2}

Profile and Soil Description

Profile description: general site information

Soil description

eutric Nitisol

Agroclimate zone

111

Location

Meru county $0^{0} 13^{\prime} \mathrm{N}, 37^{0} 47^{\prime} \mathrm{E}$

Local Petrography

Physiography

Upper Nyambene volcanic (Lava)

Relif, Macro

Hill

Land use

Hilly slope

Erosion

Under tree trials

Internal drainage

Profile description:-

Gully erosion in uncovered ground

well drained

Ah $\quad 0-40 \mathrm{~cm}$

Dark reddish brown (2.5YR 4/, dry, 2.5YR 3/4 moist) Clay:-moderate, medium subangular blocky structure, slightly hard when dry, Friable when wet, sticky and plastic when wet, Many very fine, fine medium and few coarse roots $\mathrm{pH} 6.5$ diffuse smooth transition to $\mathrm{Bu}$.

BU 40- $60 \mathrm{~cm}$

Dark reddish brown $(2.5 \mathrm{YR} 4 /$, dry, $2.5 \mathrm{YR} 3 / 4$ moist) Clay:-moderate, medium subangular blocky structure, slightly hard when dry, Friable when wet, sticky and plastic when wet, Many very fine, fine medium and few coarse roots $\mathrm{pH} 6.0$ diffuse wavy transition to $\mathrm{Bt}$

$\mathrm{Bt}_{1} 60-80 \mathrm{~cm}$

Dark reddish brown (2.5YR 3/4 moist) Clay:-moderate strong, medium sub-angular blocky structure, slightly hard when dry, Friable when wet, sticky and plastic when wet, few fine clay cutans, many very fine medium and few coarse roots $\mathrm{pH} 6.1$ clear wavy transition to $\mathrm{Bt}_{2}$.

$\mathrm{Bt}_{1} 80-120 \mathrm{~cm}$

Dark red (2.5YR 4/8, 2.5YR ,3/3 moist) Clay:-moderate to strong medium subangular blocky structure, slightly hard when dry, Friable when wet, sticky and plastic when wet, few fine clay cutans, many fine pores , few very fine and wavy few medium coarse roots $\mathrm{pH} 6.3$ 
Details of soil

\begin{tabular}{|c|c|c|c|c|c|c|c|c|}
\hline Field Designation & $\begin{array}{l}\text { No } 1 \\
\text { A }\end{array}$ & $\mathrm{B}$ & $\mathrm{C}$ & $\mathrm{D}$ & $\begin{array}{l}\text { No } 3 \\
\text { A }\end{array}$ & $\mathrm{B}$ & $\mathrm{C}$ & $\mathrm{D}$ \\
\hline Lab No.81 & 10359 & 10360 & 10361 & 10362 & 10363 & 10364 & 10365 & 10366 \\
\hline Depth & Top & $1 \mathrm{ft}$ & $2 \mathrm{ft}$. & $3 \mathrm{ft}$. & Top & $1 \mathrm{ft}$. & $2 \mathrm{ft}$. & $3 \mathrm{ft}$. \\
\hline & \multicolumn{7}{|c|}{ Chemical tests } & \\
\hline $\mathrm{pH}$ & 6.2 & 5.6 & 5.9 & 5.9 & 6.4 & 5.8 & 5.8 & 6.0 \\
\hline Na m.e. $\%$ & 0.2 & 0.1 & 0.1 & 0.13 & 0.1 & 0.1 & 0.1 & Trace \\
\hline K m.e. $\%$ & 1.52 & 1.12 & 1.16 & 0.18 & 1.42 & 0.7 & 0.5 & 0.52 \\
\hline Ca m.e. $\%$ & 8.6 & 3.0 & 3.4 & 3.0 & 8.2 & 1.7 & 0.7 & 0.4 \\
\hline Mg m.e.\% & 6.1 & 4.8 & 4.4 & 4.0 & 7.0 & 4.0 & 2.8 & 3.2 \\
\hline Mn m.e.\% & 0.66 & 0.96 & 0.86 & 0.81 & 0.81 . & 0.86 & 0.78 & 0.96 \\
\hline P p.p.m & 16 & 12 & 12 & 10 & 14 & 8 & 8 & 8 \\
\hline N \% & - & - & - & - & - & - & - & - \\
\hline \multirow[t]{2}{*}{$\mathrm{C} \%$} & 3.12 & 1.25 & & & 2.62 & 1.43 & & \\
\hline & & \multicolumn{4}{|c|}{ Soil classification } & & & \\
\hline Horizon & $\mathrm{AH}$ & $\mathrm{BU}$ & $\mathrm{BT}_{1}$ & $\mathrm{BT}_{2}$ & & & & \\
\hline Depth $(\mathrm{cm})$ & 0.40 & $40-60$ & $60-80$ & $80-120$ & & & & \\
\hline $\mathrm{pH}-\mathrm{H}_{2} \mathrm{O}\left(1=2^{1 / 2 \mathrm{~V}} / \mathrm{v}\right)$ & 6.5 & 6.0 & 6.1 & 5.3 & & & & \\
\hline pH-KCL & 5.4 & 5.1 & 5.3 & 5.5 & & & & \\
\hline $\mathrm{EC}(\mathrm{Mo} / \mathrm{cm})$ & 0.09 & 0.09 & 0.05 & 0.06 & & & & \\
\hline \multicolumn{5}{|l|}{$\mathrm{CaCO}_{3}$} & & & & \\
\hline \multicolumn{5}{|l|}{$\mathrm{CaSO}_{4}$} & & & & \\
\hline $\mathrm{C}(\%)$ & 1.26 & 0.46 & 0.69 & 0.54 & & & & \\
\hline \multicolumn{5}{|l|}{$\mathrm{N}(\%)$} & & & & \\
\hline \multicolumn{5}{|l|}{$\mathrm{C} / \mathrm{N}$} & & & & \\
\hline CEC me/100g pH 8.2 & 20.0 & 13.2 & 15.4 & 15.4 & & & & \\
\hline \multicolumn{5}{|l|}{ CEC me/100g pH 7.0} & & & & \\
\hline Exch. Ca (me/100g) & 4.8 & 2.0 & 3.9 & 4.8 & & & & \\
\hline Exch. Mg (me/100g) & 1.96 & 2.24 & 4.80 & 5.48 & & & & \\
\hline Exch. K (me/100g) & 1.98 & 0.81 & 1.25 & 1.38 & & & & \\
\hline Exch. Mn (me/100g) & 0.38 & 0.24 & 0.41 & 0.88 & & & & \\
\hline Sum of cations & 9.12 & 5.29 & 10.35 & 12.54 & & & & \\
\hline Base sat. \% pH 8.2 & 46 & 40 & 67 & 81 & & & & \\
\hline \multirow{2}{*}{\multicolumn{6}{|c|}{ Base sat. $\% \mathrm{pH} 7.0$}} & & & \\
\hline & & & & & & & & \\
\hline \multicolumn{6}{|l|}{ Gravel \% (>2.0mm) } & & & \\
\hline $\begin{array}{llll}\begin{array}{l}\text { Sand \% } \\
0.05 \mathrm{~mm})\end{array} & (2.0 & - \\
\end{array}$ & 16 & 14 & 14 & 14 & & & & \\
\hline $\begin{array}{lccc}\text { Silt } \quad \% & (0.05 & - \\
0.002 \mathrm{~mm}) & & \end{array}$ & 16 & 16 & 14 & 12 & & & & \\
\hline Clay \% $(0.002-0 \mathrm{~mm})$ & 68 & 70 & 72 & 74 & & & & \\
\hline \multirow[t]{2}{*}{ Texture class } & $\mathrm{C}$ & $\mathrm{C}$ & $\mathrm{C}$ & $\mathrm{C}$ & & & & \\
\hline & & \multicolumn{4}{|c|}{ Fertility aspect $0-30 \mathrm{~cm}$} & & & \\
\hline $\mathrm{pH}-\mathrm{H}_{2} \mathrm{O}(1 ; \mathrm{v} / \mathrm{v})$ & 6.3 & & & & & & & \\
\hline \multicolumn{6}{|l|}{$\begin{array}{ll}\text { Exch. } & \text { Acidity } \\
(\mathrm{me} / 100 \mathrm{~g})\end{array}$} & & & \\
\hline $\mathrm{C}$ & 1.29 & & & & & & & \\
\hline \multirow[t]{2}{*}{$\mathrm{N}$} & 0.15 & & & & & & & \\
\hline & & \multicolumn{4}{|c|}{ Available nutrients } & & & \\
\hline \multicolumn{6}{|l|}{$\mathrm{Na}(\mathrm{me} / 100 \mathrm{~g})$} & & & \\
\hline $\mathrm{K}(\mathrm{me} / 100 \mathrm{~g})$ & & 1.00 & & & & & & \\
\hline $\mathrm{Ca}(\mathrm{me} / 100 \mathrm{~g})$ & & 1.4 & & & & & & \\
\hline \multicolumn{6}{|l|}{$\mathrm{Mg}(\mathrm{me} / 100 \mathrm{~g})$} & & & \\
\hline $\mathrm{Mn}(\mathrm{me} / 100 \mathrm{~g})$ & & 0.66 & & & & & & \\
\hline $\mathrm{P}(\mathrm{ppm})$ & & 12 & & & & & & \\
\hline P-Olsen (ppm) & & & & & & & & \\
\hline
\end{tabular}

